IUCrJ

ISSN 2052-2525

MATERIALS|COMPUTATION

Received 10 February 2020

Accepted 10 March 2020

Edited by P. Lightfoot, University of St Andrews, Scotland

Keywords: lone pairs; chemical bonding; thermal conductivity; quantum crystallography; electron densities.

Supporting information: this article has supporting information at www.iucrj.org

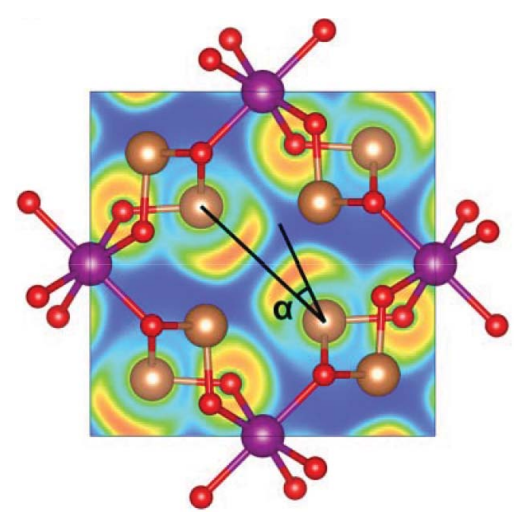

OPEN $\odot$ ACCESS

\section{Expression and interactions of stereochemically active lone pairs and their relation to structural distortions and thermal conductivity}

\author{
Kasper Tolborg, ${ }^{a}$ Carlo Gatti ${ }^{\mathrm{b}}$ and Bo B. Iversen ${ }^{\mathrm{a} *}$
}

${ }^{a}$ Center for Materials Crystallography, Department of Chemistry and iNANO, Aarhus University, Langelandsgade 140, Aarhus 8000, Denmark, and ${ }^{\mathbf{b} C N R-S C I T E C}$ Istituto di Scienze e Tecnologie Chimiche 'Giulio Natta', via Golgi Section, via Golgi 19, Milano 20133, Italy. *Correspondence e-mail: bo@chem.au.dk

In chemistry, stereochemically active lone pairs are typically described as an important non-bonding effect, and recent interest has centred on understanding the derived effect of lone pair expression on physical properties such as thermal conductivity. To manipulate such properties, it is essential to understand the conditions that lead to lone pair expression and provide a quantitative chemical description of their identity to allow comparison between systems. Here, density functional theory calculations are used first to establish the presence of stereochemically active lone pairs on antimony in the archetypical chalcogenide $\mathrm{MnSb}_{2} \mathrm{O}_{4}$. The lone pairs are formed through a similar mechanism to those in binary post-transition metal compounds in an oxidation state of two less than their main group number [e.g. $\mathrm{Pb}$ (II) and $\mathrm{Sb}$ (III)], where the degree of orbital interaction (covalency) determines the expression of the lone pair. In $\mathrm{MnSb}_{2} \mathrm{O}_{4}$ the $\mathrm{Sb}$ lone pairs interact through a void space in the crystal structure, and their their mutual repulsion is minimized by introducing a deflection angle. This angle increases significantly with decreasing $\mathrm{Sb}-\mathrm{Sb}$ distance introduced by simulating high pressure, thus showing the highly destabilizing nature of the lone pair interactions. Analysis of the chemical bonding in $\mathrm{MnSb}_{2} \mathrm{O}_{4}$ shows that it is dominated by polar covalent interactions with significant contributions both from charge accumulation in the bonding regions and from charge transfer. A database search of related ternary chalcogenide structures shows that, for structures with a lone pair ( $\mathrm{Sb} X_{3}$ units), the degree of lone pair expression is largely determined by whether the antimony-chalcogen units are connected or not, suggesting a cooperative effect. Isolated $\mathrm{Sb} X_{3}$ units have larger $X-\mathrm{Sb}-X$ bond angles and therefore weaker lone pair expression than connected units. Since increased lone pair expression is equivalent to an increased orbital interaction (covalent bonding), which typically leads to increased heat conduction, this can explain the previously established correlation between larger bond angles and lower thermal conductivity. Thus, it appears that for these chalcogenides, lone pair expression and thermal conductivity may be related through the degree of covalency of the system.

\section{Introduction}

Stereochemically active lone pairs are usually treated as textbook examples of non-bonding effects and occur in posttransition metal compounds in which the post-transition metal is in an oxidation state of two lower than its main group number, such as $\mathrm{Pb}$ (II) and $\mathrm{Sb}(\mathrm{III})$. This means that the two outermost $s$ electrons are available to form a lone pair, leading to, after hybridization, the possibility of an asymmetric coordination environment. These oxidation states are found in many technologically important materials, such as thermoelectric materials (Snyder \& Toberer, 2008; Zhao et al., 2014), multiferroics (Ramesh \& Spaldin, 2007), phase-change materials (Lencer et al., 2008) and optoelectronics (Ogo et al., 2008; 
Zhou et al., 2015). But their structural chemistry is quite intriguing, since the ability to form a lone pair does not always lead to asymmetric coordination, e.g. in symmetric rock salt $\mathrm{PbS}$ and asymmetric litharge $\mathrm{PbO}$ (Walsh \& Watson, 2005b). Originally, and still in most textbooks, stereochemically active lone pairs are described as on-site $s p$ hybridization on the metal atom, which is the origin of its name as a 'chemically inactive' (i.e. non-bonding) but 'stereochemically active' (i.e. structure-determining) effect. However, this does not fully explain the anion dependence on the tendency to form an asymmetric coordination environment.

In a series of articles, Walsh, Watson and co-workers introduced a revised model to account for this anion dependence based also on work by Waghmare and co-workers (Walsh et al., 2011; Watson et al., 1999; Watson \& Parker, 1999; Walsh \& Watson, 2005a,b; Waghmare et al., 2003). They showed that the lone pairs are in fact not chemically inactive, but rather that the $s$ orbital on the metal atom mixes with the anion $p$ orbital to form a bonding and an anti-bonding state, followed by mixing of the anti-bonding state with the metal $p$ states. The tendency to form a lone pair is therefore highly dependent on the energy difference between the metal valence states and the anion valence $p$ states. If this difference is large, the formation of the bonding orbital is less favourable, and thus the lone pair is not 'expressed' and the higher symmetry structure is adopted (Walsh et al., 2011). Thus, the expression of a lone pair is highly dependent on covalent interactions between cation and anion.

With these trends established, we have a framework for understanding the structures of materials with the possibility of forming stereochemically active lone pairs. The relation to physical properties has been investigated for thermoelectric materials, where the presence of oxidation states, which are able to form stereochemically active lone pairs, was shown to lead to a decrease in lattice thermal conductivity in the $\mathrm{Cu}-$ $\mathrm{Sb}-\mathrm{Se}$ ternary system, i.e. $\mathrm{Sb}^{3+}$ systems have lower thermal conductivity than $\mathrm{Sb}^{5+}$ systems (Skoug \& Morelli, 2011). Furthermore, an empirical correlation between bond angle and lattice thermal conductivity in $\mathrm{As}, \mathrm{Sb}$ and Bi chalcogenides in oxidation state +3 was established with larger bond angles generally leading to lower thermal conductivity. The ability to form a lone pair has also been used to describe the good electronic properties for thermoelectricity of rock salt lead and tin monochalcogenides compared with other rock salt materials (Zeier et al., 2016). Interestingly, it is often not the formation of a stereochemically active lone pair, but merely the presence of the investigated oxidation states that result in favourable thermoelectric properties; in some cases, the expression of a lone pair is even detrimental for either electronic or thermal properties in closely related systems (Cagnoni et al., 2018; Yu et al., 2020).

However, the general relation to physical properties and the crystal structures beyond the asymmetric local coordination environment is still poorly understood. The structural point is illustrated by the large difference in crystal structures adopted by otherwise similar materials, where the local coordination environment is asymmetric, such as the localized molecular

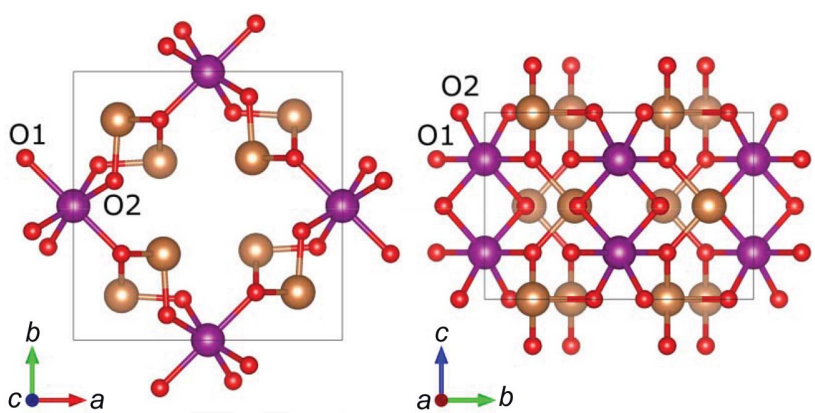

Figure 1

Crystal structure of $\mathrm{MnSb}_{2} \mathrm{O}_{4}$ viewed along the $c$ axis (left) and $a$ axis (right). $\mathrm{Mn}$ is shown as purple, $\mathrm{Sb}$ as brown and $\mathrm{O}$ as red. The two crystallographically unique oxygen atoms are marked as $\mathrm{O} 1$ and $\mathrm{O} 2$.

units in $\mathrm{Sb}_{2} \mathrm{O}_{3}$ and the infinite chains in $\mathrm{Sb}_{2} \mathrm{~S}_{3}$. Similarly, the bond angles around the stereochemically active lone pair can be very different for otherwise similar systems (Wang \& Liebau, 1996; Skoug \& Morelli, 2011).

An interesting chalcogenide material in which the local coordination environment is asymmetric is the isostructural group of ternary oxides $M \mathrm{Sb}_{2} \mathrm{O}_{4}$, where $M$ is a transition metal. Structures have been reported for $M=\mathrm{Mn}, \mathrm{Fe}, \mathrm{Co}, \mathrm{Ni}$ and $\mathrm{Zn}$, crystallizing in the space group $P 4_{2} / m b c$, isostructural to red lead, $\mathrm{Pb}_{3} \mathrm{O}_{4}$, which can formally be written as $\mathrm{Pb}(\mathrm{IV}) \mathrm{Pb}(\mathrm{II})_{2} \mathrm{O}_{4}$ to highlight the similarity between the groups. The crystal structure of $\mathrm{MnSb}_{2} \mathrm{O}_{4}$ is shown in Fig. 1 along the $c$ and $a$ axes. The structure consists of distorted $\mathrm{MnO}_{6}$ octahedra with two long $\mathrm{Mn}-\mathrm{O} 1$ and four short $\mathrm{Mn}-$ $\mathrm{O} 2$ distances, and $\mathrm{SbO}_{3}$ units in a trigonal pyramidal coordination with two long $\mathrm{Sb}-\mathrm{O} 1$ and one short $\mathrm{Sb}-\mathrm{O} 2$ distances, leaving room for a presumed lone pair on antimony to occupy the fourth corner in a tetrahedron (Müller-Buschbaum, 2003; Roelsgaard et al., 2016).

The $M \mathrm{Sb}_{2} \mathrm{O}_{4}$ compounds are relatively unexplored in the literature, but they have been studied for potential use in $\mathrm{Li}$ ion batteries due to presence of channels of low electron density along the $c$ axis, and magnetic structures have been studied and were shown to result in different orderings depending on the metal atom (Fjellvåg et al., 1985; Jibin et al., 2012; Roelsgaard et al., 2016; Nørby et al., 2016). Our present interest in this class of materials arises from the interesting structure, where the presumed position of the antimony lone pair based on the coordination environment suggests that two lone pairs point almost directly towards each other. Basic chemical intuition would suggest that this interaction should be highly unfavourable, and similar structural motifs are not, to our knowledge, adopted by other materials with stereochemically active lone pairs.

Here we investigate the expression and interactions between the presumed stereochemically active lone pairs on antimony in $\mathrm{MnSb}_{2} \mathrm{O}_{4}$ through density functional theory (DFT) calculations based on both orbital and electron density related descriptors to gain further understanding of this intriguing structural motif. Furthermore, we simulate the highpressure behaviour of the material to force the lone pairs 
closer together and investigate the response of this external stimulus. Then, we report a characterization of all other bonding interactions in the material, and finally, we perform a database analysis on a group of related structures in order to derive general features regarding the influence of stereochemically active lone pairs on crystal structures, and their relation to physical properties, particularly thermal conductivity.

\section{Computational details}

Periodic ab initio DFT calculations on $\mathrm{MnSb}_{2} \mathrm{O}_{4}$ were performed in CRYSTAL14 using the POB-TZVP basis set (Dovesi et al., 2014; Laun et al., 2018; Peintinger et al., 2013). This basis set uses a full-potential all-electron basis for $\mathrm{Mn}$ and $\mathrm{O}$ and a small-core effective core potential for $\mathrm{Sb}$ with 23 electrons in the valence corresponding to the $4 s^{2} 4 p^{6} 4 d^{10} 5 s^{2} 5 p^{3}$ electrons. The PBE0 hybrid functional was used and reciprocal space was sampled on an $8 \times 8 \times 8$ grid in a Monkhorst-Pack net in the first Brillouin zone (Adamo \& Barone, 1999; Perdew et al., 1996). The initial geometry and magnetic symmetry were set to the geometry from Roelsgaard et al. (2016) and the antiferromagnetic configuration corresponding to the A-mode from Fjellvåg et al. (1985). In principle, this results in lowering the space group symmetry to $P \overline{4} b 2$, which would require displacement of all atomic $z$ coordinates by $1 / 4$ to follow the standard settings of the space groups. However, in this case it was achieved using the CRYSTAL14 keyword MODISYMM to remove (half of) the symmetry elements to maintain the coordinates corresponding to those in the structural space group. A ferromagnetic configuration was also tried, but resulted in higher energy and similar bonding features.

First, the full geometry, i.e. cell and atomic coordinates, was optimized with the symmetry from the magnetic structure. The resulting atomic coordinates still followed the structural space group symmetry within the numerical error. Results of the optimization are given in the supporting information. Here it is seen that the deviation of the optimized geometry from the experimental structure at $100 \mathrm{~K}$ is less than $0.5 \%$ for cell parameters and less than $2 \%$ for bond lengths. After this, a series of unit-cell volumes from $10 \%$ smaller to $4 \%$ larger than the equilibrium volume in steps of $2 \%$ were constructed and the cell parameters and atomic coordinates were relaxed at constant volume (CVOLOPT keyword). The energy-volume curve was fitted to a third-order Birch-Murnaghan equationof-state to find the corresponding pressures.

The chemical bonding was analysed in terms of projected density of states and valence electron density as implemented in CRYSTAL14, and topological analysis following Bader's Quantum Theory of Atoms in Molecules (QTAIM) (Bader, 1990) using TOPOND interfaced with CRYSTAL14 (Gatti et al., 1994).

\section{Results and discussion}

\subsection{Density of states and valence electron density}

To establish the presence of a stereochemically active lone pair on antimony, we first investigate the orbital projected density of states (DOS) and the valence electron density in the region of interest. The DOS (Fig. 2) can be divided into four distinct regions in accordance with previously established stereochemically active lone pairs in $\mathrm{Sb}_{2} \mathrm{O}_{3}$ and tin monochalcogenides (Allen et al., 2013; Walsh \& Watson, 2005a). Region IV is special for this material compared with, for example, $\mathrm{Sb}_{2} \mathrm{O}_{3}$ due to the transition metal and consists mainly of manganese $d$ states and oxygen $p$ states. A similar feature is also seen in region III, but here we also observe a large degree of overlap between oxygen $p$ states and antimony $s$ and $p$ states. Further below the Fermi level, we have region II, which consists mainly of oxygen $p$ states and antimony $p$ states, and region I, which consists mainly of antimony $s$ states and oxygen $p$ states. Regions I, II and III are qualitatively very similar to the ones in, for example, $\mathrm{Sb}_{2} \mathrm{O}_{3}$, with the complication of the presence of a transition metal here (Allen et al., 2013).

To further highlight the character of these regions, we can plot the valence electron density within these energy intervals. The most interesting regions regarding the stereochemically active lone pair are regions I and III, which are shown in Fig. 3. In Figs. 3(a) and 3(b), we see that region I consists of a bonding interaction between $\mathrm{Sb}$ and $\mathrm{O}$, both to $\mathrm{O} 1$ and to $\mathrm{O} 2$. However, we clearly observe that the electron density in this energy range is higher for the long $\mathrm{Sb}-\mathrm{O} 1$ bond. This oxygen is bonded to two $\mathrm{Sb}$ and one $\mathrm{Mn}$, which seems to affect the energy levels of its valence states. This indicates that the interaction between $\mathrm{Sb}$ and $\mathrm{O} 1$ is the dominating feature for the expression of the lone pair.

In region III [Figs. $3(c)$ and $3(d)$ ] the lone pair on $\mathrm{Sb}$ and localized electron density on $\mathrm{O}$ are clearly observed. This region is identified as the key for the stereochemically active lone pair, and this valence density is in very good correspondence with the density observed in, for example, SnO by Walsh \& Watson (2005a), which is one of the archetypical examples of stereochemically active lone pairs induced by the anion. Thus, it is safe to conclude that the present material has stereochemically active lone pairs on antimony that follow the

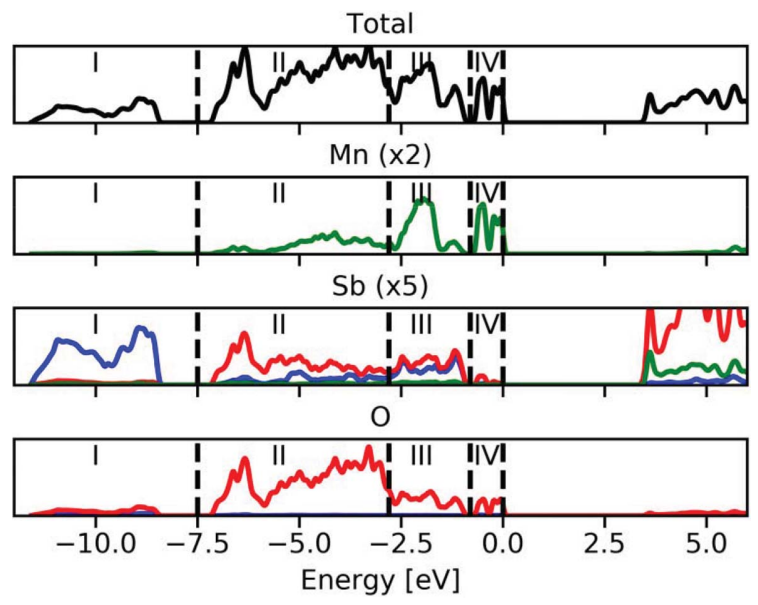

Figure 2

Total and orbital projected electronic density of states. Blue lines are $s$ states, red lines are $p$ states and green lines are $d$ states. Individual atomic contributions have been enlarged to allow visual inspection. 


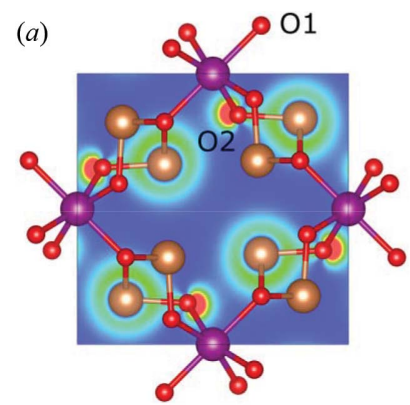

(b)

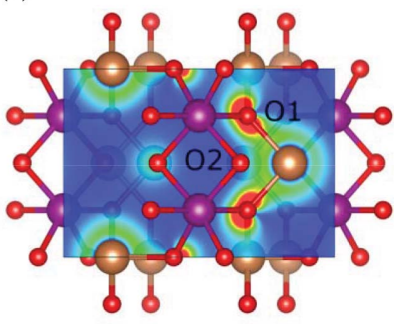

(c)

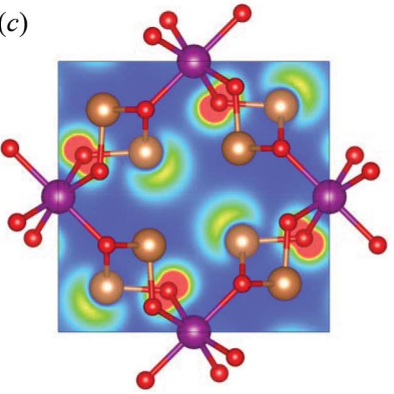

(d)

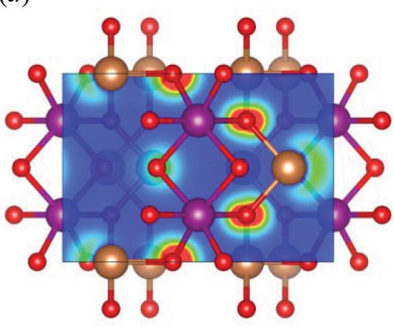

Figure 3

Valence electron density in selected planes and regions from Fig. 2. $(a)$ and $(b)$ Region I, $(c)$ and $(d)$ Region III. $(a)$ and $(c)(001)$ plane at $z=$ $1 / 2,(b)$ and $(d)$ plane spanned by $\mathrm{Sb}$ and two equivalent $\mathrm{O} 1$ atoms. Contours are drawn from 0 (blue) to $0.0445 \mathrm{e} \mathrm{bohr}^{-3}$ (red). Mn is shown as purple, $\mathrm{Sb}$ as brown and $\mathrm{O}$ as red, and the two crystallographically unique $\mathrm{O}$ atoms are again marked as $\mathrm{O} 1$ and $\mathrm{O} 2$ in $(a)$ and $(b)$. The figures were generated using VESTA (Momma \& Izumi, 2008).

established framework, although with some complexity induced by the presence of the transition metal causing, for example, the different oxygen atoms to behave quite differently.

\subsection{Real space identification of the lone pairs}

So far, we have considered the electronic structure from an orbital projected point of view. However, it is interesting also to analyse the lone pairs and the chemical bonding from the perspective of real space descriptors, assessing, for example, the electron localization. A lone pair is characterized by a large degree of electron localization, and it is thus commonly identified using descriptors that assess the localization of electrons or the concentration of the electron density. The two commonly used descriptors are the electron localization function (ELF) (Becke \& Edgecombe, 1990; Silvi \& Savin, 1994; Savin et al., 1997) and the Laplacian of the electron density, $\nabla^{2} \rho(r)$ (Gatti, 2005), respectively.

In Fig. 4(a), the ELF is plotted in the (001) plane at $z=1 / 2$, i.e. at the plane containing the two antimony atoms pointing towards each other. The electrons appear to be extremely localized at the expected lone pair region on antimony; furthermore, we see a tendency of the lone pairs to avoid each other as the maximum localizations are located at a significant angle away from the interatomic line, i.e. angle $\alpha$ in Fig. 4(a). It is also interesting to note that not only do the ELF maxima tend to avoid each other, but the lone pairs are themselves asymmetric. This leads to alignment of high and low electron

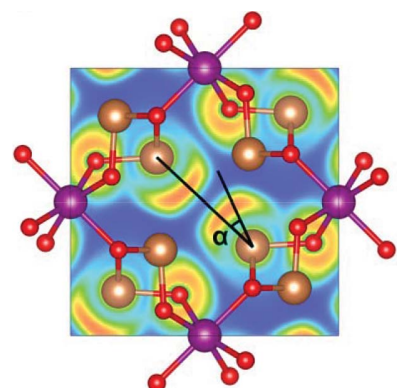

(a)

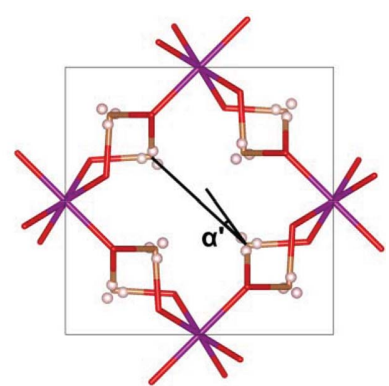

(b)
Figure 4

(a) ELF in the (001) plane at $z=1 / 2$. Contours are drawn from 0 (blue) to 1 (red). (b) Maxima in $L(r)=-\nabla^{2} \rho(r)$ in the $\mathrm{N}$-shell of $\mathrm{Sb}$. The deviation of the lone pair maxima from the interatomic line in the (a) ELF and $(b)$ $L(r)$ are indicated by the angles $\alpha$ and $\alpha^{\prime}$, respectively. Mn is shown as purple, $\mathrm{Sb}$ as brown and $\mathrm{O}$ as red.

localizations, which minimizes the unfavourable interactions between the lone pairs.

In Fig. 4(b), the lone pair is visualized using the Laplacian of the electron density. If plotting it in a 2D plane like the ELF, no obvious features are seen, so instead, we search for critical points in the charge concentration on antimony. Typically, one would perform the search in the outermost valence shell, but for heavy atoms, this is often buried within the charge depletions of the inner shells (Shi \& Boyd, 1988). Therefore, we use the procedure outlined by Sist et al. (2017), who showed that the lone pair character is not only present as a charge concentration in the outermost valence shell, but also in the shell below. In Fig. 4(b), the four charge concentrations in the $N$-shell on $\mathrm{Sb}$ are shown, and they correspond well to an $s p^{3}$ hybridization with three concentrations pointing towards neighbouring oxygen atoms, and one charge concentration pointing in the direction where the lone pairs were also observed from the ELF. Also, from the charge concentrations, a slight deviation from the interatomic line is observed as indicated by the angle $\alpha^{\prime}$.

\subsection{Pressure effects on lone pair interaction}

We have now shown that there is a destabilizing interaction due to lone pairs pointing towards each other, which is presumably decreased by introducing an angle between the lone pairs. To further investigate this feature, we simulated the effect of pressure on the structure by optimizing the structure at various constant volumes and extracting the pressure from the energy-volume relation. In Fig. 5, the unit-cell parameters and bond lengths are shown as a function of pressure. We can see that the $a$ axis contracts more with increased pressure, which makes sense since the channels of low electron density run along the $c$ axis, meaning that there is a void space for the structure to relax into in the $a b$ plane. Interestingly, we see that the bond lengths change significantly with pressure, where the originally short $\mathrm{Mn}-\mathrm{O} 2$ bond remains almost constant with pressure, whereas the $\mathrm{Mn}-\mathrm{O} 1$ bond length decreases significantly with pressure. Eventually, the order of the bond lengths switches at a very moderate pressure between 1 and $2 \mathrm{GPa}$. This is a consequence of the $\mathrm{Mn}-\mathrm{O} 1$ bond lying in the 
Table 1

Bond critical points and properties evaluated at these points.

$R$ is distance between the atoms; $d 1$ and $d 2$ are the distances from the BCP to the first and second atom, respectively; $\rho\left(r_{\mathrm{b}}\right)$ and $\nabla^{2} \rho\left(r_{\mathrm{b}}\right)$ are the electron density and Laplacian of the electron density; $G\left(r_{\mathrm{b}}\right), V\left(r_{\mathrm{b}}\right)$ and $H\left(r_{\mathrm{b}}\right)$ are the kinetic, potential and total energy densities at the BCP; and $\varepsilon$ is the ellipticity. The Sb-O2 bond marked by an asterisk is not shown in the structural figures, as it is significantly longer than the typical bond length.

\begin{tabular}{|c|c|c|c|c|c|c|c|c|c|c|}
\hline & $R(\AA)$ & $d 1(\AA)$ & $d 2(\AA)$ & $\begin{array}{l}\rho\left(r_{\mathrm{b}}\right) \\
\left(\mathrm{e} \AA^{-3}\right)\end{array}$ & $\begin{array}{l}\nabla^{2} \rho\left(r_{\mathrm{b}}\right) \\
\left(\mathrm{e} \AA^{-5}\right)\end{array}$ & $\begin{array}{l}G\left(r_{\mathrm{b}}\right) \\
\left(\text { Hartree } \AA^{-3}\right)\end{array}$ & $\begin{array}{l}V\left(r_{\mathrm{b}}\right) \\
\left(\text { Hartree } \AA^{-3}\right)\end{array}$ & $\begin{array}{l}H\left(r_{\mathrm{b}}\right) \\
\left(\text { Hartree } \AA^{-3}\right)\end{array}$ & $\begin{array}{l}G\left(r_{\mathrm{b}}\right) / \rho\left(r_{\mathrm{b}}\right) \\
\left(\text { Hartree }^{-1}\right)\end{array}$ & $\varepsilon$ \\
\hline $\mathrm{Mn}-\mathrm{O} 2$ & 2.16 & 1.07 & 1.09 & 0.39 & 6.13 & 0.50 & -0.57 & -0.07 & 1.27 & 0.03 \\
\hline $\mathrm{M}-\mathrm{O} 1$ & 2.18 & 1.08 & 1.10 & 0.35 & 5.69 & 0.45 & -0.50 & -0.05 & 1.26 & 0.04 \\
\hline $\mathrm{Sb}-\mathrm{O} 1$ & 2.02 & 1.03 & 0.99 & 0.70 & 9.01 & 0.83 & -1.02 & -0.20 & 1.19 & 0.05 \\
\hline $\mathrm{Sb}-\mathrm{O} 2 *$ & 2.84 & 1.49 & 1.35 & 0.14 & 1.33 & 0.09 & -0.09 & 0.00 & 0.67 & 0.01 \\
\hline $\mathrm{Sb}-\mathrm{Sb}$ & 4.22 & 2.11 & 2.11 & 0.04 & 0.27 & 0.02 & -0.01 & 0.00 & 0.38 & 0.08 \\
\hline
\end{tabular}

$a b$ plane, which contracts the most. Here it should be noted that the difference between the $\mathrm{Mn}-\mathrm{O} 1$ and $\mathrm{Mn}-\mathrm{O} 2$ bond lengths at zero pressure is smaller in our optimization than from experiment at $100 \mathrm{~K}$, so experimentally the switch may not occur until at a higher pressure (see Table S1 of the supporting information). For the $\mathrm{Sb}-\mathrm{O}$ bonds, the changes are less significant, but interestingly enough the short $\mathrm{Sb}-\mathrm{O} 2$ bond, which lies in the $a b$ plane, actually increases slightly as a function of pressure, whereas the longer $\mathrm{Sb}-\mathrm{O} 1$ bond
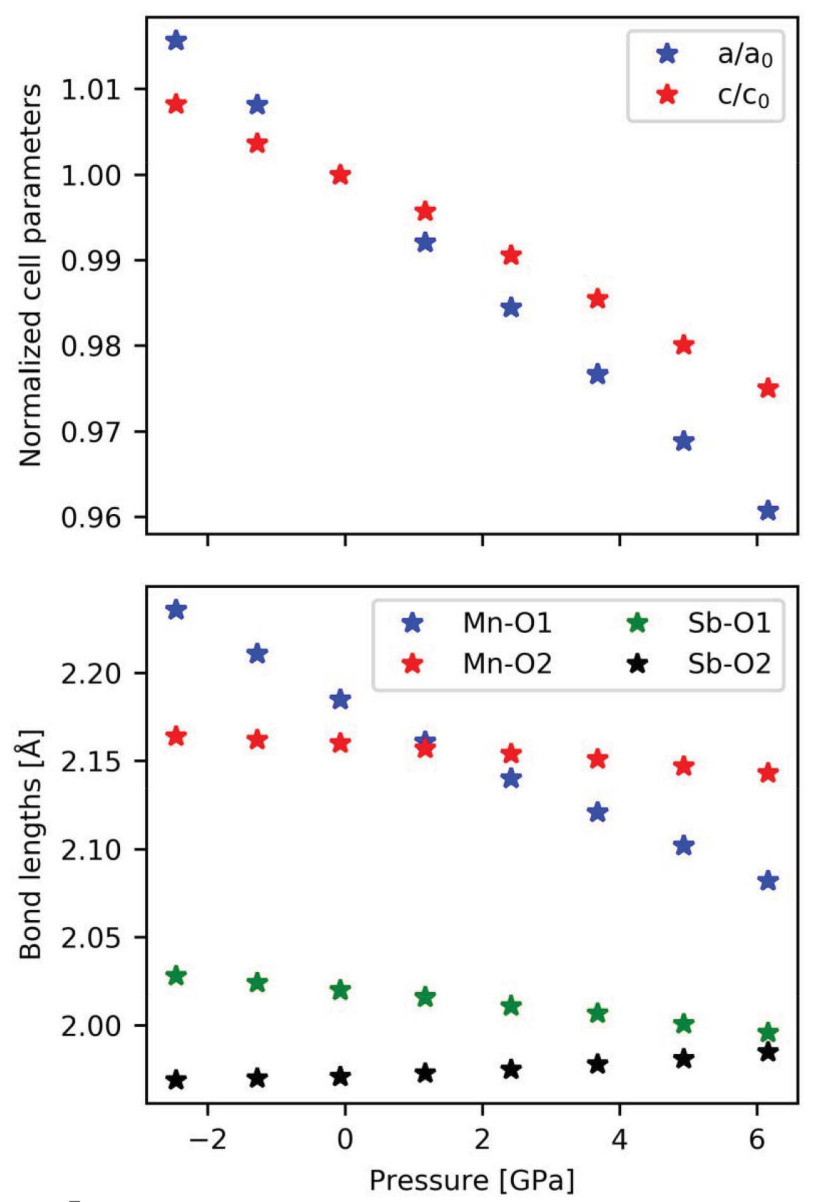

Figure 5

Normalized cell parameters and bond lengths of the four unique short bonds as a function of pressure. The optimized geometry used in the previous section is seen to be at slightly low negative pressure from a Birch-Murnaghan third-order fit to the energy-volume curve. decreases slightly. It is assumed that no phase transition occurs, although a phase transition has been observed for the iron analogue, $\mathrm{FeSb}_{2} \mathrm{O}_{4}$, at around $4 \mathrm{GPa}$ (Hinrichsen et al., 2006).

In Fig. 6(a), the effect of pressure on the lone pair deflection gauged by the maxima in $L(r)=-\nabla^{2} \rho(r)$ is shown [angle $\alpha^{\prime}$ in Fig. 4(b)]. It is clear that, with decreasing distance between $\mathrm{Sb}$ atoms resulting from the high pressure, the angle between the interatomic vector and the lone pair vector increases. The enhanced angle with increasing pressure (and a shorter $\mathrm{Sb}-$ $\mathrm{Sb}$ distance) is also qualitatively visualized in Figs. $6(b)$ and $6(c)$, where the ELF is seen at a volume reduced by 6 and $10 \%$, respectively, to enable comparison with the ELF for the optimized volume shown in Fig. 4(a). This deflection is a clear effect of the repulsion between lone pairs, which use the flexibility of the structure to reduce the repulsion as much as possible.

\subsection{Topological analysis of the electron density}

Having analysed the interactions between the lone pairs on antimony, we now report the general bonding scheme in the material. Since the electron density distribution is a quantum mechanical observable, it is useful to analyse the chemical bonding (almost) purely based on this, which is the foundation of Bader's QTAIM (Bader, 1990). Here we define the topological atom based on partitioning of the electron density using the zero-flux surface, and we define a bonding interaction based on the $(3,-1)$ critical points, i.e. points where the density is at the minimum along one direction (parallel to the bond) and the maximum along the other two directions (perpendicular to the bond). Based on the properties at the bond critical point (BCP), we can obtain valuable information about chemical bonding based on well established relations that can often be related to structural and physical properties (Gatti, 2005; Tolborg \& Iversen, 2019).

In Table 1, all BCPs are shown. BCPs are found at all four of the unique bonds drawn in Fig. 1, i.e. two unique $\mathrm{Mn}-\mathrm{O}$ and two unique $\mathrm{Sb}-\mathrm{O}$ bonds. In addition, BCPs are found between $\mathrm{Sb}$ and $\mathrm{O} 2$ at a larger distance and between the two $\mathrm{Sb}$ atoms 'through' the lone pair. All bonds have quite low, but non-negligible density and a positive Laplacian, which is, however, not surprising since the BCPs lie in a region of 

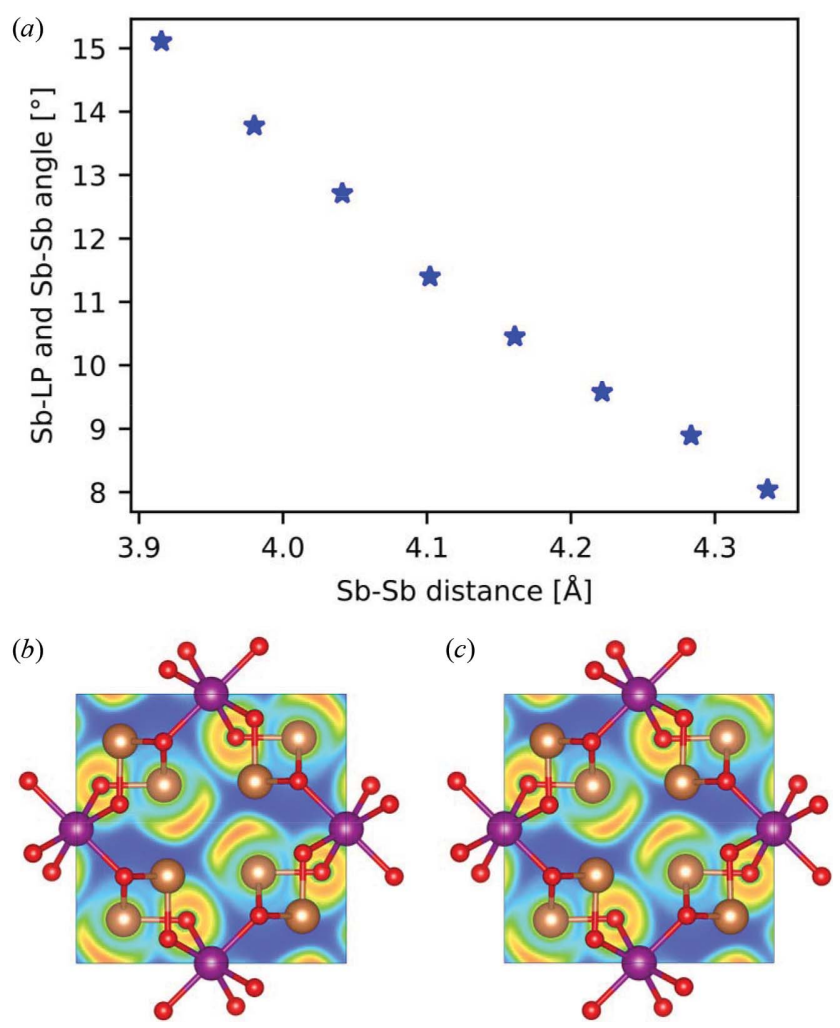

Figure 6

(a) Angle between the $\mathrm{Sb}$ to lone pair vector and the $\mathrm{Sb}$ to $\mathrm{Sb}$ vector as a function of pressure. The angle is indicated as $\alpha^{\prime}$ in Fig. 4(b) for the equilibrium volume. $(b)$ and $(c)$ ELF in the (001) plane at $z=1 / 2$ for the structure with a volume reduction of $(b) 6 \%$ and $(c) 10 \%$. Contours are drawn from 0 (blue) to 1 (red); (b) and (c) are compared with Fig. 4(a) at equilibrium volume.

charge depletion on the heavy atoms and at a distance from the nuclei where the charge distribution of the corresponding isolated atoms is also depleted (Shi \& Boyd, 1988). Looking at the energy densities, we see that all four short bonds have negative total energy densities, and generally the shorter the bond, the larger the electron density and the more negative the total energy density. Furthermore, the kinetic energy per electron, $G\left(r_{\mathrm{b}}\right) / \rho\left(r_{\mathrm{b}}\right)$, is slightly above one. All these characteristics are common for polar covalent bonds and donoracceptor bonds (Gatti, 2005). The integrated atomic charges in Table 2 show that large ionic contributions are present in all interactions, since charges are found to be +1.53 and +1.89 on $\mathrm{Mn}$ and Sb, respectively. These charges are quite high, showing the large degree of ionic bonding, although significantly smaller than those corresponding to their formal oxidation states.

In Fig. 7, the deformation density and negative Laplacian of the electron density are shown for selected planes. Here, we observe that there is some charge accumulation in all four types of short bonds. It is especially interesting to note that manganese shows positive deformation density towards oxygen, which clearly highlights the covalent contribution to this bond. In the $\mathrm{Sb}-\mathrm{O}$ bonds, the deformation density is less pronounced and both positive and negative deformation density are observed along the bond path. This is in very good
Table 2

Integrated atomic properties.

Atomic charges $(Q)$ are in units of electrons, volumes $(V)$ in $\AA^{3}$ and integrated Lagrangian $(L)$ in atomic units. Only the unique atoms from the structural point of view are shown. The only numerically significant differences between magnetically different atoms are the spin density related properties.

\begin{tabular}{lrcr}
\hline & $Q$ & $V$ & \multicolumn{1}{c}{$L$} \\
\hline $\mathrm{Mn}$ & 1.53 & 9.47 & $-9.1 \times 10^{-3}$ \\
$\mathrm{Sb}$ & 1.89 & 23.0 & $2.1 \times 10^{-3}$ \\
$\mathrm{O} 1$ & -1.30 & 14.3 & $1.5 \times 10^{-4}$ \\
$\mathrm{O} 2$ & -1.35 & 14.3 & $3.3 \times 10^{-4}$ \\
\hline
\end{tabular}

agreement with the observation from both theory and experiment in $\mathrm{Sb}_{2} \mathrm{O}_{3}$ (Whitten et al., 2004). Combined with the fact that charge concentrations on antimony point towards oxygen, this shows that there is some degree of covalency in this bonding, meaning that the $\mathrm{Sb}-\mathrm{O}$ bonds are of polar covalent type.

Another reasonable interpretation of the chemical bonding would be in terms of charge-shift bonding (Shaik et al., 2009; Shaik et al., 2020). Here the important contribution to the bond energy is the resonance energy between ionic and covalent valence bond (electronic) structures, and this is generally found to lead to a small, but non-negligible electron density and a positive Laplacian at the BCP. For light maingroup elements, the distinction between covalent and chargeshift bonding is easily made, since a negative Laplacian is expected for a (polar) covalent bond. In the present case, since a negative Laplacian is not expected for a (polar) covalent bond involving heavy elements, we cannot unequivocally assign these bonds to either polar covalent or charge-shift bonds without resorting to a valence bond model wavefunction analysis. In either case, there is a significant degree of covalency and therefore orbital overlap in the chemical bonding, which is important for reconciling the orbital-based view with our analysis of the electron density, since the theory of stereochemically active lone pairs requires significant orbital overlap.

The two extra BCPs found are between atoms at a much longer distance, the density is much lower and the total energy density is practically zero. This shows that they are largely present due to the geometry dictated by the stronger interactions, rather than being important structure-determining features on their own. The $\mathrm{Sb}-\mathrm{Sb} \mathrm{BCP}$ in particular is clearly not a stabilizing interaction, since we have shown that the charge concentrations try to avoid each other to lower the repulsion between adjacent lone pairs, rather than directing them towards each other as seen in typical covalent bonds.

\subsection{Implications for other materials with stereochemically active lone pairs}

The clear repulsion between stereochemically active lone pairs, which we have demonstrated here, might have important implications for our understanding of crystal structures with expressed stereochemically active lone pairs. In almost all other structures, an important structural motif is that lone 


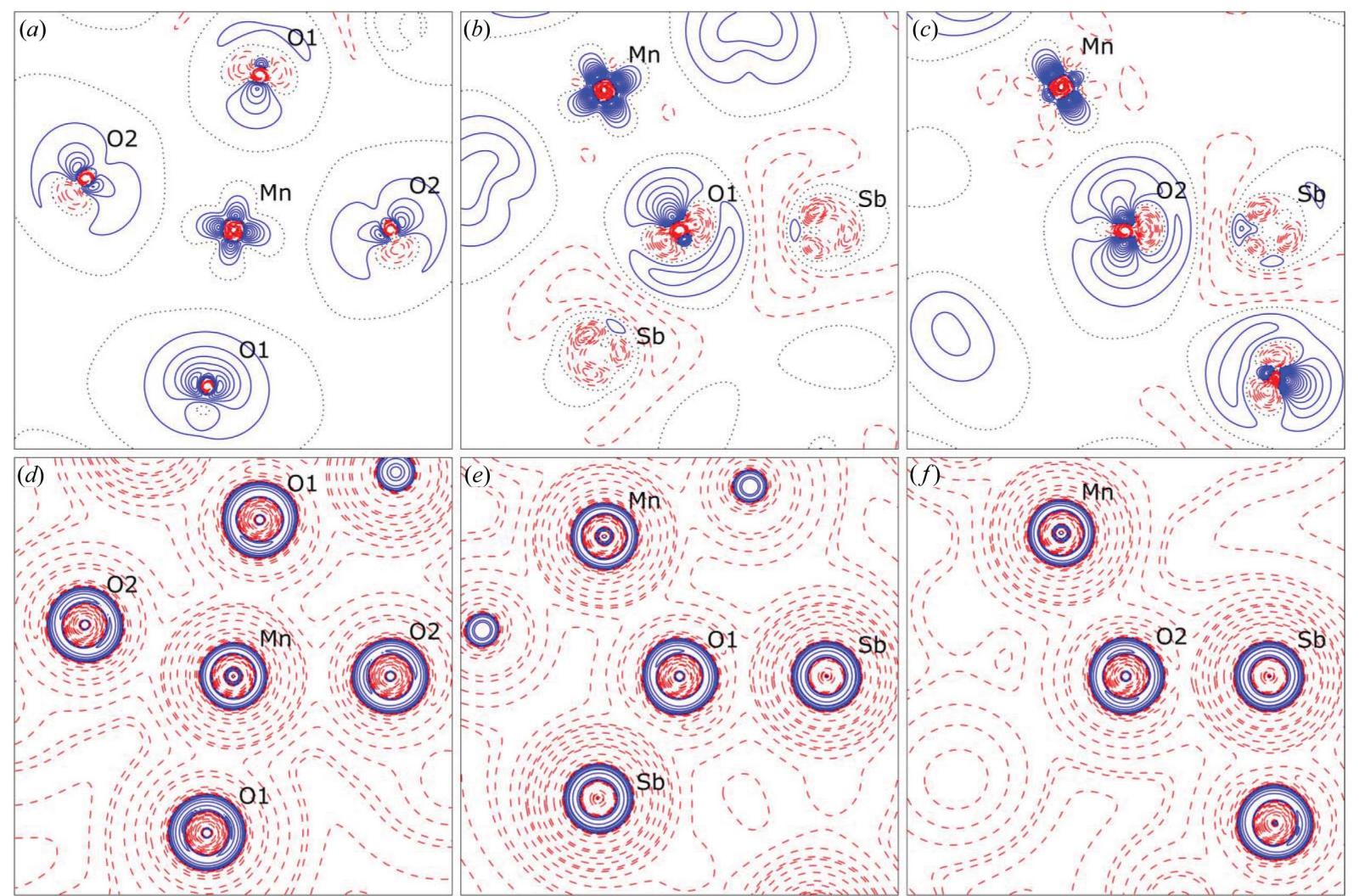

Figure 7

(a)-(c) Deformation densities in the planes containing the labelled nuclei; positive (solid blue), negative (dashed red) and zero (dotted black) contours are shown and contour spacings are 0.1 e $\AA^{-3}$ in $(a)$ and 0.05 e $\AA^{-3}$ in $(b)$ and $(c)$. $(d)-(f)$ Negative Laplacian of the electron density in selected planes; positive (solid blue), negative (dashed red) and zero (dotted black) contours are shown at $a \times 10^{n}$, where $a=1,2,4,8$ and $n=-2,-1,0,1,2,3,4$.

pairs point into areas where no other lone pairs are present such as in the litharge structure adopted by $\mathrm{SnO}$ and $\mathrm{PbO}$, and the GeS structure adopted by GeS, GeSe, $\mathrm{SnS}$ and SnSe. Even in a structure like cubic $\mathrm{Sb}_{2} \mathrm{O}_{3}$ (senarmonite), where the lone pair region points towards other $\mathrm{Sb}$ atoms, they arrange in a tetrahedron to avoid having charge concentrations pointing directly towards each other (Walsh et al., 2011). In the present structure, the lone pairs are forced into the same region in pairs, but tend to decrease this unfavourable interaction by introducing a deflection angle. Furthermore, the lone pairs only interact strongly between pairs of atoms, but each of these atomic pairs tend to occupy mutually exclusive regions of space to decrease interactions with other atomic pairs, similarly to the individual behaviour in the other structures mentioned. Thus, the repulsive nature of the lone pairs has an important structure determining role, which often leads to the formation of structural motifs such as layers, channels or cages.

As mentioned earlier, there are indications that the unique oxygen atom bonded to two antimony atoms was much more involved in the lone pair formation than the other, which is only bonded to one $\mathrm{Sb}$ atom. This suggests that there could be a cooperative effect involved in the lone pair expression. Skoug \& Morelli (2011) showed an empirical correlation between bond angles and lattice thermal conductivity in chalcogenides of $\mathrm{As}, \mathrm{Sb}$ and $\mathrm{Bi}$. In this case, bond angles were used as a measure for the degree of lone pair expression according to valence shell electron pair repulsion (VSEPR) theory, since a more strongly expressed lone pair closer to the nuclei will give rise to smaller bond angles. In their example case of the $\mathrm{Cu}-\mathrm{Sb}-\mathrm{Se}$ system, the main structural difference between the two materials $\mathrm{CuSbSe}_{2}$ and $\mathrm{Cu}_{3} \mathrm{SbSe}_{3}$, where the lone pair is more strongly expressed in the former, is that the $\mathrm{SbSe}_{3}$ units are connected in $\mathrm{CuSbSe}_{2}$, but isolated in $\mathrm{Cu}_{3} \mathrm{SbSe}_{3}$. These two observations motivated us to perform a database search in the Inorganic Crystal Structure Database (ICSD) (Bergerhoff et al., 1983) in order to understand whether it is a general trend that isolated units tend to have larger bond angles arising from weaker lone pair expression, and therefore possibly lower lattice thermal conductivity as a result. The search included all $M \mathrm{Sb}$ (III) $X$ structures (where $M$ is an alkali metal, an alkaline-earth metal or a transition metal, and $X$ is $\mathrm{O}, \mathrm{S}$ or $\mathrm{Se}$ ) from the ICSD, where the coordination number of antimony is three. Structures with partial occupancies (i.e. disorder) and structures where the atomic coordinates were not refined were excluded. The structures were sorted based on whether the $\mathrm{Sb} X_{3}$ units were isolated or connected. In order to make this distinction, an operational definition of a bond, which depends only on the geometry, must be used. We tried a criterion based on covalent radii, but to include a reasonable number of bonds an arbitrary increase of the sum of covalent radii must be used. Instead we chose to use a criterion based on bond valence parameters, $s=\exp \left[\left(r_{0}\right.\right.$ 
- $r) / B]$, where $B=0.37$ is a universal constant, $r_{0}$ is the tabulated bond valence parameter, $r$ is the bond length and $s$ is the corresponding bond valence (Brown \& Altermatt, 1985; Brese \& Keeffe, 1991). It was suggested by Altermatt \& Brown (1985) that values larger than 0.6 for the bond valence correspond to a covalent bond, and values larger than $0.038 \times$ the oxidation state of the cation correspond to a bond to be included in the calculation of the effective valence. However, these two numbers are too strict and too loose, respectively, in the present case for a reasonable number of bonds that should be included, so we chose a bond valence value of 0.3 to be the minimum value for a bond to be included in the analysis. This corresponds to maximum distances of 2.42, 2.90 and $3.02 \AA$ for bonds between $\mathrm{Sb}$ and $\mathrm{O}, \mathrm{S}$ and $\mathrm{Se}$, respectively. Changing the parameters slightly makes a small difference in the final histogram, but does not affect the conclusions.

Fig. 8 shows that, despite a significant degree of overlap between the two groups, there is a clear tendency for isolated $\mathrm{Sb} X_{3}$ units to have larger bond angles than the connected ones. According to VSEPR theory, this means that the lone pair is further from the nuclei in the isolated cases, or put differently, the lone pair is less expressed. Therefore, the present results suggest that there is a cooperative effect involved in the stronger expression of the lone pair, meaning the ability of the chalcogen to be involved in lone pair expression becomes stronger when it is influenced by more than one $\mathrm{Sb}$ atom. Since the bond angles, and thus lone pair expression, are strongly correlated with lattice thermal conductivity, this gives an interesting handle for designing new low thermal conductivity structures with potential applications as thermoelectric materials.

As discussed above, lone pair expression arises from an orbital interaction, i.e. a covalent chemical interaction, so the

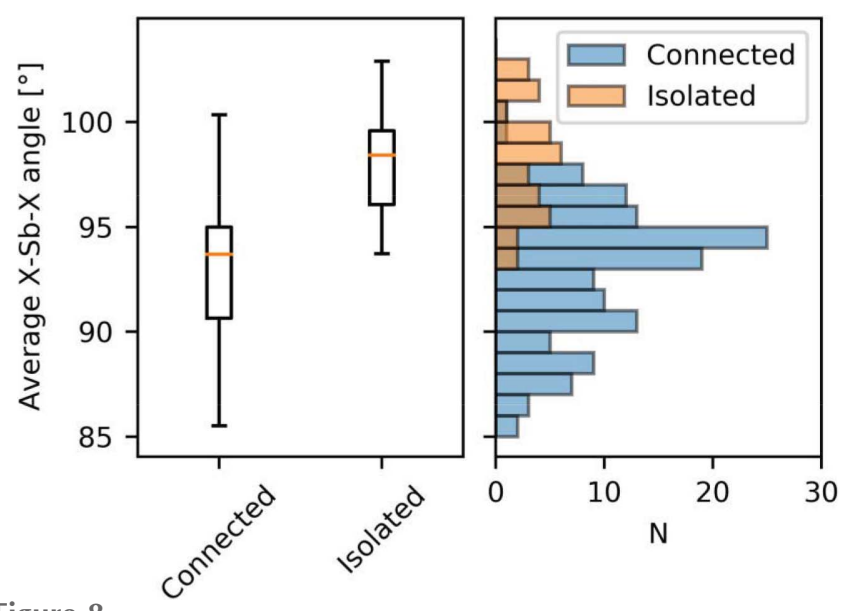

Figure 8

Average $X-\mathrm{Sb}-X$ angle for $M S \mathrm{Sb}(\mathrm{III}) X$ structures (where $M$ is an alkali metal, an alkaline earth metal or a transition metal, and $X$ is $\mathrm{O}, \mathrm{S}$ or Se) from the ICSD, depending on whether the $\mathrm{Sb} X_{3}$ units are connected or isolated. To the left a box plot is shown with an orange bar marking the median value, the box marks the interquartile range and the whiskers mark the spread of the data points. To the right a histogram shows the number of $\mathrm{Sb} X_{3}$ units $(N)$ with bond angles within the given interval. Data are binned in $1^{\circ}$ intervals. The search is based on 77 crystal structures with 137 unique connected $\mathrm{Sb} X_{3}$ units and 35 unique isolated $\mathrm{Sb} X_{3}$ units. See Table S2 for a list of structures and ICSD codes. larger degree of lone pair expression should correspond to a more covalent interaction. It is well known that stronger bonding leads to larger phonon velocities and therefore larger lattice thermal conductivity (Slack, 1973). This also means that heat conduction mainly occurs along covalent bonds, which was quantified for a layered system by Zhang et al. (2018), who found a strong correlation between the electron density at the bond critical points and the thermal conductivity in presumably layered structures. Thus, we would expect that weaker orbital interaction should lead to lowering of the thermal conductivity. Therefore, an explanation for the lower lattice thermal conductivity in systems with larger bond angles shown by Skoug \& Morelli (2011) may arise from weaker covalent interactions in these systems, originating from whether the units are connected or isolated, and indeed, they showed that $\mathrm{Cu}_{3} \mathrm{SbSe}_{3}$ with isolated $\mathrm{Sb} X_{3}$ units has lower thermal conductivity than $\mathrm{CuSbSe}_{2}$ with connected units.

Previously, the differences in bond angles have been interpreted as differences in effective atomic valence of the $\mathrm{Sb}$ atom (Wang \& Liebau, 1996). Here a perfect tetrahedron with four neighbours (bond angles of $109.5^{\circ}$ ) corresponds to an oxidation state of +5 and a complete transfer of the $5 s$ lone pair to a bonding interaction with an anion, whereas an oxidation state of +3 in a threefold coordination corresponds to a completely localized lone pair and thus a small bond angle. Intermediate cases then correspond to a progressive change in effective oxidation state and a less localized lone pair, which results in a larger bond angle. This was based on the fact that shorter bond lengths, and thus formally a larger effective valence, were observed for larger bond angles. Alternatively, one can imagine the limit of a flat coordination with $120^{\circ}$ angles corresponding to an $s p^{2}$-hybridized $\mathrm{Sb}$ with a lone pair in the remaining $p$ orbital. Progressive changes towards small bond angles correspond to more $s$ character in the lone pair, and correspondingly more $p$ character in the $\mathrm{Sb}-X$ bonds, which leads to longer bonds, similar to the increase in the $\mathrm{C}-\mathrm{H}$ bond lengths with increasing $p$ character in going from acetylene $(s p)$ through ethylene $\left(s p^{2}\right)$ to ethane $\left(s p^{3}\right)$ (Bent, 1961), and in agreement with previous observations (Wang \& Liebau, 1996). Interestingly, it is the smaller bond angles corresponding to the longer bonds that give rise to the largest thermal conductivity. Also in the case of $\mathrm{MnSb}_{2} \mathrm{O}_{4}$, it is in fact the longer $\mathrm{Sb}-\mathrm{O}$ bond that has the largest degree of covalency determined from the valence electron densities and is more involved in lone pair formation. The present group of materials is thus a counterexample to the otherwise established correlation between shorter bond lengths and higher thermal conductivity, which is present over a very wide range of bond lengths and thermal conductivities, but with several groups of similar systems not following the trend (Zeier et al., 2016). Thus, it seems to be the covalency of an interaction, rather than the bond length itself, which is important for the thermal conductivity.

The lattice thermal conductivity of a material is determined from several contributions, which in simple terms can be divided into two groups: those arising from the phonon dispersion itself (e.g. phonon group velocity) and those arising 
from the scattering of phonons (e.g. phonon-phonon scattering from anharmonicity or impurity scattering). Lone pair expression is often discussed in terms of the presence of asymmetric coordination, and the repulsion between the lone pair and valence electrons on neighbouring atoms, which is thought to lead to an anharmonic vibration potential, and therefore a decreased thermal conductivity through phononphonon scattering (Skoug \& Morelli, 2011; Zhang et al., 2012). With the present observations of increased covalency for connected units and the correspondence between lone pair expression and covalency, we instead suggest that lone pair expression and thermal conductivity are mainly related through the covalency of the system, and not necessarily because of specific anharmonic potential directions. This means that for weakly expressed lone pairs, the smaller degree of covalency should give rise to lower phonon velocities and a less rigid electronic structure, leading to lower thermal conductivity.

An interesting limiting case for our discussion is structures with a coordination number of six in perfect octahedral symmetry such as the rock salt lead chalcogenides, where the lone pair is not expressed in the static structure. During atomic displacement, the lone pair will be weakly expressed in different spatial regions depending on the direction of vibration, corresponding to a large coupling between atomic displacement and electronic structure. This leads to dynamic local distortions in this type of materials (Bozin et al., 2010; Sangiorgio et al., 2018). The bonding in these materials has been classified as resonant bonding (Lencer et al., 2008; Shportko et al., 2008), and this has been suggested as the origin of their low thermal conductivity by leading to phonon softening and strong anharmonicity (Lee et al., 2014). More recently, the concept was termed metavalent bonding, and it was shown that displacement of GeTe along its Peierls distortion is associated with more covalent bonding and a more rigid electronic structure showed by a decrease in response properties such as the Born effective charge (Raty et al. 2019). This distortion can be seen as a transition starting in the ideal symmetric rock-salt structure, where simple electron counting and the electron sharing calculated by Raty et al. (2019) reveal the formation of six 2-centre-1-electron (2c-1e) bonds between $\mathrm{Ge}$ and $\mathrm{Te}$ formed by their $p$ orbitals, while the remaining valence electrons are localized on the atoms in the spherically symmetric $s$ orbitals. Upon displacement of Ge along the [111] direction, three short and three long bonds are formed. In the completely distorted limit, the bonding situation approaches three $2 \mathrm{c}-2 \mathrm{e}$ bonds. In this case, due to the loss of symmetry, the last two electrons on Ge will no longer be distributed spherically and symmetrically, but rather form an expressed lone pair as the last corner in a tetrahedron, resembling the bonding situation in GeSe (Sist et al., 2017). Thus, the consequence of approaching covalent bonding from metavalent bonding in a system like GeTe is also expression of a stereochemically active lone pair.

In this way, the relation between bond angle and thermal conductivity found by Skoug \& Morelli (2011) can be considered as a progressive change from covalent bonding (strong lone pair expression, small bond angle) towards metavalent or resonant bonding (weak lone pair expression, large bond angle), which leads to lowering of the thermal conductivity. Interestingly, as we have shown here, the tendency to form small or large bond angles is strongly influenced by whether the $\mathrm{Sb} X_{3}$ units are connected or isolated.

The strong coupling between atomic displacement and electronic structure may give rise to anharmonicity, for example, in thermoelectric $\mathrm{SnSe}$ where a specific anharmonic mode revealed from inelastic neutron scattering was shown to highly perturb the electronic structure in the regions of interest for the lone pair ( $\mathrm{Li}$ et al., 2015). Similarly, anharmonicity has also been revealed both experimentally and theoretically in rock salt lead and tin chalcogenides (Delaire et al., 2011; Li et al., 2014; Lee et al., 2014; Shulumba et al., 2017). However, strong anharmonicity is not a necessary criterion for obtaining low thermal conductivity, and it is also important to acknowledge that the degree of covalency in a system will be important in determining its lattice thermal conductivity. It is clearly of interest to further probe relations between the extent of lone pair expression, thermal conductivity, structural disorder and anharmonic thermal motion.

\section{Conclusions}

We have shown that $\mathrm{MnSb}_{2} \mathrm{O}_{4}$ has all the characteristics of a material with $\mathrm{Sb}(\mathrm{III})$ stereochemically active lone pairs. We observe significant contribution of antimony $5 s$ and $5 p$ states close to the Fermi level, and from the valence density, this is attributed to an antibonding configuration expressed as a stereochemically active lone pair. From real space descriptors such as the ELF and the Laplacian of the electron density, we found the positions of the antimony lone pairs, which were shown to avoid each other by inducing an angle between the lone pair and the interatomic line. This deflection was shown to increase with decreasing distance between antimony atoms by simulating the effect of pressure on the material. The chemical bonding in the material was shown to have a significant degree of covalency, which is important for our understanding of the lone pair formation, as the current theory requires a significant degree of orbital overlap. Analysis of the valence electron density suggested that the oxygen atom bonded to two $\mathrm{Sb}$ atoms was more involved in lone pair formation than the one bonded to only one $\mathrm{Sb}$ atom. This inspired a database search for structures with isolated and connected $\mathrm{Sb} X_{3}$ units (where $X$ is a chalcogen), and showed that larger bond angles are generally found for isolated units. These observations suggest a degree of cooperative effect in the lone pair expression. Since heat conduction is normally largest along covalent bond directions, a stronger lone pair expression should lead to higher thermal conductivity, as is indeed the case for $\mathrm{Sb} X_{3}$ structures. Thus, it appears that for these chalcogenides, lone pair expression and thermal conductivity may be mainly related through the degree of covalency of the system, which affects the phonon dispersion, and not necessarily through strong anharmonicity, which 
decreases the thermal conductivity via phonon-phonon scattering.

\section{Funding information}

This work was supported by the Danish National Research foundation (grant No. DNRF93) and the Villum Foundation. The theoretical calculations were performed at the Center for Scientific Computing, Aarhus. Affiliation with the Aarhus University Center for Integrated Materials Research (iMAT) is gratefully acknowledged.

\section{References}

Adamo, C. \& Barone, V. (1999). J. Chem. Phys. 110, 6158-6170.

Allen, J. P., Carey, J. J., Walsh, A., Scanlon, D. O. \& Watson, G. W. (2013). J. Phys. Chem. C, 117, 14759-14769.

Altermatt, D. \& Brown, I. D. (1985). Acta Cryst. B41, 240-244.

Bader, R. F. W. (1990). Atoms in Molecules: A Quantum Theory. Oxford: Clarendon Press.

Becke, A. D. \& Edgecombe, K. E. (1990). J. Chem. Phys. 92, 53975403.

Bent, H. A. (1961). Chem. Rev. 61, 275-311.

Bergerhoff, G., Hundt, R., Sievers, R. \& Brown, I. D. (1983). J. Chem. Inf. Model. 23, 66-69.

Bozin, E. S., Malliakas, C. D., Souvatzis, P., Proffen, T., Spaldin, N. A., Kanatzidis, M. G. \& Billinge, S. J. L. (2010). Science, 330, 1660-1663.

Brese, N. E. \& O'Keeffe, M. (1991). Acta Cryst. B47, 192-197.

Brown, I. D. \& Altermatt, D. (1985). Acta Cryst. B41, 244-247.

Cagnoni, M., Führen, D. \& Wuttig, M. (2018). Adv. Mater. 30, 1801787.

Delaire, O., Ma, J., Marty, K., May, A. F., McGuire, M. A., Du, M. H., Singh, D. J., Podlesnyak, A., Ehlers, G., Lumsden, M. D. \& Sales, B. C. (2011). Nat. Mater. 10, 614-619.

Dovesi, R., Orlando, R., Erba, A., Zicovich-Wilson, C. M., Civalleri, B., Casassa, S., Maschio, L., Ferrabone, M., De La Pierre, M., D'Arco, P., Noël, Y., Causà, M., Rérat, M. \& Kirtman, B. (2014). Int. J. Quantum Chem. 114, 1287-1317.

Fjellvåg, H. \& Kjekshus, A. (1985). Acta Chem. Scand. A 39, 389-395. Gatti, C. (2005). Z. Krist. Cryst. Mater. 220, 399-457.

Gatti, C., Saunders, V. R. \& Roetti, C. (1994). J. Chem. Phys. 101, 10686-10696.

Hinrichsen, B., Dinnebier, R. E., Rajiv, P., Hanfland, M., Grzechnik, A. \& Jansen, M. (2006). J. Phys. Condens. Matter, 18, S1021-S1037.

Jibin, A. K., Reddy, M. V., Subba Rao, G. V., Varadaraju, U. V. \& Chowdari, B. V. R. (2012). Electrochim. Acta, 71, 227-232.

Laun, J., Vilela Oliveira, D. \& Bredow, T. (2018). J. Comput. Chem. 39, 1285-1290.

Lee, S., Esfarjani, K., Luo, T. F., Zhou, J. W., Tian, Z. T. \& Chen, G. (2014). Nat. Commun. 5, 3525.

Lencer, D., Salinga, M., Grabowski, B., Hickel, T., Neugebauer, J. \& Wuttig, M. (2008). Nat. Mater. 7, 972-977.

Li, C. W., Hong, J., May, A. F., Bansal, D., Chi, S., Hong, T., Ehlers, G. \& Delaire, O. (2015). Nat. Phys. 11, 1063-1069.

Li, C. W., Ma, J., Cao, H. B., May, A. F., Abernathy, D. L., Ehlers, G., Hoffmann, C., Wang, X., Hong, T., Huq, A., Gourdon, O. \& Delaire, O. (2014). Phys. Rev. B, 90, 214303.

Momma, K. \& Izumi, F. (2008). J. Appl. Cryst. 41, 653-658.

Müller-Buschbaum, H. (2003). J. Alloys Compd. 349, 49-104.

Nørby, P., Roelsgaard, M., Søndergaard, M. \& Iversen, B. B. (2016). Cryst. Growth Des. 16, 834-841.
Ogo, Y., Hiramatsu, H., Nomura, K., Yanagi, H., Kamiya, T., Hirano, M. \& Hosono, H. (2008). Appl. Phys. Lett. 93, 032113.

Peintinger, M. F., Oliveira, D. V. \& Bredow, T. (2013). J. Comput. Chem. 34, 451-459.

Perdew, J. P., Burke, K. \& Ernzerhof, M. (1996). Phys. Rev. Lett. 77, 3865-3868.

Ramesh, R. \& Spaldin, N. A. (2007). Nat. Mater. 6, 21-29.

Raty, J. Y., Schumacher, M., Golub, P., Deringer, V. L., Gatti, C. \& Wuttig, M. (2019). Adv. Mater. 31, 1806280.

Roelsgaard, M., Nørby, P., Eikeland, E., Søndergaard, M. \& Iversen, B. B. (2016). Dalton Trans. 45, 18994-19001.

Sangiorgio, B., Bozin, E. S., Malliakas, C. D., Fechner, M., Simonov, A., Kanatzidis, M. G., Billinge, S. J. L., Spaldin, N. A. \& Weber, T. (2018). Phys. Rev. Mater. 2, 085402.

Savin, A., Nesper, R., Wengert, S. \& Fassler, T. F. (1997). Angew. Chem. Int. Ed. 36, 1809-1832.

Shaik, S., Danovich, D., Galbraith, J. M., Braïda, B., Wu, W. \& Hiberty, P. C. (2020). Angew. Chem. Int. Ed. 59, 984-1001.

Shaik, S., Danovich, D., Wu, W. \& Hiberty, P. C. (2009). Nat. Chem. 1, 443-449.

Shi, Z. \& Boyd, R. J. (1988). J. Chem. Phys. 88, 4375-4377.

Shportko, K., Kremers, S., Woda, M., Lencer, D., Robertson, J. \& Wuttig, M. (2008). Nat. Mater. 7, 653-658.

Shulumba, N., Hellman, O. \& Minnich, A. J. (2017). Phys. Rev. B, 95, 014302.

Silvi, B. \& Savin, A. (1994). Nature, 371, 683-686.

Sist, M., Gatti, C., Nørby, P., Cenedese, S., Kasai, H., Kato, K. \& Iversen, B. B. (2017). Chem. Eur. J. 23, 6888-6895.

Skoug, E. J. \& Morelli, D. T. (2011). Phys. Rev. Lett. 107, 235901.

Slack, G. A. (1973). J. Phys. Chem. Solids, 34, 321-335.

Snyder, G. J. \& Toberer, E. S. (2008). Nat. Mater. 7, 105-114.

Tolborg, K. \& Iversen, B. B. (2019). Chem. Eur. J. 25, 15010-15029.

Waghmare, U. V., Spaldin, N. A., Kandpal, H. C. \& Seshadri, R. (2003). Phys. Rev. B, 67, 125111.

Walsh, A., Payne, D. J., Egdell, R. G. \& Watson, G. W. (2011). Chem. Soc. Rev. 40, 4455-4463.

Walsh, A. \& Watson, G. W. (2005a). J. Phys. Chem. B, 109, 1886818875.

Walsh, A. \& Watson, G. W. (2005b). J. Solid State Chem. 178, 14221428.

Wang, X. \& Liebau, F. (1996). Acta Cryst. B52, 7-15.

Watson, G. W. \& Parker, S. C. (1999). J. Phys. Chem. B, 103, $1258-$ 1262.

Watson, G. W., Parker, S. C. \& Kresse, G. (1999). Phys. Rev. B, 59, 8481-8486.

Whitten, A. E., Dittrich, B., Spackman, M. A., Turner, P. \& Brown, T. C. (2004). Dalton Trans. 23-29.

Yu, Y., Cagnoni, M., Cojocaru-Mirédin, O. \& Wuttig, M. (2020). Adv. Funct. Mater. 30, 1904862.

Zeier, W. G., Zevalkink, A., Gibbs, Z. M., Hautier, G., Kanatzidis, M. G. \& Snyder, G. J. (2016). Angew. Chem. Int. Ed. 55, 68266841.

Zhang, J. W., Song, L. R., Sist, M., Tolborg, K. \& Iversen, B. B. (2018). Nat. Commun. 9, 4716.

Zhang, Y. S., Skoug, E., Cain, J., Ozoliņš, V., Morelli, D. \& Wolverton, C. (2012). Phys. Rev. B, 85, 054306.

Zhao, L. D., Lo, S. H., Zhang, Y. S., Sun, H., Tan, G. J., Uher, C., Wolverton, C., Dravid, V. P. \& Kanatzidis, M. G. (2014). Nature, 508, 373-377.

Zhou, Y., Wang, L., Chen, S. Y., Qin, S. K., Liu, X. S., Chen, J., Xue, D. J., Luo, M., Cao, Y. Z., Cheng, Y. B., Sargent, E. H. \& Tang, J. (2015). Nat. Photon. 9, 409-415. 